\title{
Design Consideration in the Development of Multi-Fin FETs for RF Applications
}

\author{
Peijie Feng, Prasanta Ghosh \\ Department of Electrical Engineering and Computer Science, Syracuse University, Syracuse, USA \\ Email: pfeng@syr.edu
}

Received March 7, 2012; revised April 17, 2012; accepted May 16, 2012

\begin{abstract}
In this paper, we propose multi-fin FET design techniques targeted for RF applications. Overlap and underlap design configuration in a base FinFET are compared first and then multi-fin device (consisting of transistor unit up to 50) is studied to develop design limitations and to evaluate their effects on the device performance. We have also investigated the impact of the number of fins (up to 50) in multi-fin structure and resulting RF parameters. Our results show that as the number of fin increases, underlap design compromises RF performance and short channel effects. The results provide technical understanding that is necessary to realize new opportunities for RF and analog mixed-signal design with nanoscale FinFETs.
\end{abstract}

Keywords: FinFET; Analog; RF; Source/Drain Extension Region Engineering; Simulation; Multi-Fin FET

\section{Introduction}

According to the International Technology Roadmap for Semiconductors (ITRS), as transistor dimension scaling into nanometer regime, the conventional planar bulk MOSFET technology faces many challenges: e.g., the close proximity between source and drain worsens leakage current; the necessary high doping in the bulk causes threshold voltage fluctuation, etc. [1]. FinFET, emerging as a promising device, addresses those Short Channel Effects (SCEs) and secures the necessary performance in the sub-32 nm regime due to its scalability, superior SCEs, and compatibilities to the planar CMOS platform.

Our survey reveals that recent papers are more on FinFET's digital application and less on analog/RF figures of merit (FoM) [2]. Several papers present work on source/ drain extension (SDE) region engineering with the goal of improving a single-fin FET or coupling FinFETs performance $\left(N_{\text {FinfET }} \leq 5\right)$ [3-5]. Only few papers are on ana$\log /$ RF FoM of multi-fin FETs which introduces a large total channel width to achieve high transconductance, maintain good noise and mismatch performance [2].

In this paper, with extensive calibrated TCAD simulations, we present results for SCEs and analog/RF FoM of a base FinFET unit and then a multi-fin FET $\left(N_{\text {FinFET }}\right.$ up to 50). The effect of SDE engineering on the multi-fin device RF performance is studied. Simulations along with theoretical analysis establish the realistic application potential of underlap design for the multi-Fin FET RF operation.

\section{Simulation Setup and Results}

\subsection{Base FinFET Unit}

Given that base FinFET units within the multi-fin configuration are nominal identical to each other, we first optimize analog/RF FoM of a $22 \mathrm{~nm}$ node single-fin FET which then will be used as a base transistor for a multifin structure [6]. The gate length $\left(L_{\mathrm{g}}\right)$ of the n-type FinFET is set at $25 \mathrm{~nm}$. The fin height $\left(H_{\mathrm{fin}}\right)$ is fixed at $50 \mathrm{~nm}$. The bulk is lightly doped $10^{15} \mathrm{~cm}^{-3}$ to avoid the dopant fluctuation. Selective epitaxial growth with heavy doping are performed for the source/drain region to minimize the parasitic resistance. SDE region engineering is considered by the application of overlap and underlap design. Abrupt junction, which is achievable by solid re-growth and laser annealing process [5], is designed with a fast doping decay with lateral straggle $\left(\sigma_{\mathrm{S} / \mathrm{D}}\right)$ at the value of 1 $\mathrm{nm} / \mathrm{dev}$ at the edge of SDE region whereas the underlap doping profile in the SDE region is simulated with a Gaussian model rolling off from a peak value of $10^{20}$ $\mathrm{cm}^{-3}$ at the edge of the source/drain. The equivalent oxide thickness (EOT) of the Hf-based dielectric in the simulation is $0.7 \mathrm{~nm}$. The work-function of the TiN metal gate is adjusted to $4.6 \mathrm{eV}$ such that the threshold voltage $V_{\mathrm{t}}$ of the device is around $0.3 \mathrm{~V}$. The device is investigated with a calibrated TCAD simulation taking into account quantum effect with Lombardi mobility model [7].

The design of TCAD experiments shown in Table 1 for this study considers the trade-off between current drivability and SCEs. Overlap design enhances current 
Table 1. Tcad-predicted SCEs of the 22-nm node DG nFin FET at room temperature for SDE engineering.

\begin{tabular}{ccccccc}
\hline $\begin{array}{c}W_{\text {fin }} \\
(\mathrm{nm})\end{array}$ & $\begin{array}{c}L_{\text {ext }} \\
(\mathrm{nm})\end{array}$ & $\begin{array}{c}\sigma_{\mathrm{S} D} \\
(\mathrm{~nm} / \mathrm{dec})\end{array}$ & $\begin{array}{c}\mathrm{DIBL} \\
(\mathrm{mV} / \mathrm{V})\end{array}$ & $\begin{array}{c}\mathrm{S} \\
(\mathrm{mV})\end{array}$ & $\begin{array}{c}I_{\text {off }} \\
(\mathrm{A} / \mu \mathrm{m})\end{array}$ & $\begin{array}{c}I_{\text {on }} \\
(\mathrm{A} / \mu \mathrm{m})\end{array}$ \\
\hline 17 & 10 & Overlap & 146 & 101.2 & $6.2 \mathrm{e}-7$ & $1.2 \mathrm{e}-3$ \\
17 & 10 & 5 & 85 & 76.1 & $3.9 \mathrm{e}-10$ & $9.1 \mathrm{e}-4$ \\
17 & 20 & Overlap & 154 & 99.4 & $7.8 \mathrm{e}-7$ & $1.2 \mathrm{e}-3$ \\
17 & 20 & 10 & 72 & 74.3 & $2.0 \mathrm{e}-9$ & $7.6 \mathrm{e}-4$ \\
12 & 10 & Overlap & 73 & 77.9 & $2.6 \mathrm{e}-9$ & $1.1 \mathrm{e}-3$ \\
12 & 10 & 5 & 34 & 66.2 & $6.9 \mathrm{e}-11$ & $8.4 \mathrm{e}-4$ \\
12 & 20 & Overlap & 77 & 76.4 & $3.2 \mathrm{e}-9$ & $1.0 \mathrm{e}-3$ \\
12 & 20 & 10 & 29 & 65.6 & $4.4 \mathrm{e}-11$ & $6.7 \mathrm{e}-4$ \\
\hline
\end{tabular}

drive at the cost of SCEs due to the S/D encroachment into the channel. Thin $W_{\text {fin }}$ can alleviate the SCEs but it degrades the drive current since the SDE resistance is increased. Underlap design specified by spacer length to lateral doping gradient ratio $\left(L_{\text {ext }} / \sigma_{\mathrm{S} / \mathrm{D}}\right)$ keeps a good compromise between the drain current and SCEs, and shows great potential in SDE region engineering [3-5]. It shows a larger intrinsic gain $\left(A_{\mathrm{VO}}\right)$ than overlap one and a comparable $g_{\mathrm{m}} / I_{\mathrm{ds}}$ ratio when $L_{\mathrm{ext}} / \sigma_{\mathrm{S} / \mathrm{D}}=2$ (See Figure 1 (a)). As $L_{\mathrm{ext}} / \sigma_{\mathrm{S} / \mathrm{D}}$ goes beyond 2 and $L_{\mathrm{ext}}$ increases, $A_{\mathrm{vo}}$ rises but $g_{\mathrm{m}} / I_{\mathrm{ds}}$ decreases quickly. The degradation is also found in Figure 1(b), where a longer $L_{\text {ext }}$ and a larger $\sigma_{\mathrm{S} / \mathrm{D}}$ bring about a poorer $g_{\mathrm{m}} / I_{\mathrm{ds}}$ ratio for different $\sigma_{\mathrm{S} / \mathrm{D}}$ devices with a fixed $L_{\text {ext }} / \sigma_{\mathrm{S} / \mathrm{D}}$. These degradations are due to increase of the undoped portion in SDE region that extends the effective channel length [3], indicating an increase of $1 / g_{\mathrm{ds}}$ as demonstrated in the same figure. Figure 1(c) shows the analog/RF FoM extracted at 100 $\mu \mathrm{A} / \mu \mathrm{m}$. Both the available $S_{21}$ (at $10 \mathrm{GHz}$ ) and intrinsic cut off frequency $\left(f_{\mathrm{T}}\right)$ reaches maximum when $L_{\mathrm{ext}} / \sigma_{\mathrm{SD}}=$ 2. Considering the fabrication fluctuation and compromise among $g_{\mathrm{m}}, A_{\mathrm{vo}}, f_{\mathrm{T}}$ and SCEs, the optimal value of $L_{\text {ext }} / \sigma_{\mathrm{SD}}$ ratio for the base FinFET is set at 2 with a $20 \mathrm{~nm}$ spacer length.

\subsection{Multi-Fin FETs}

For a single-fin FET, boosting of $g_{\mathrm{m}}$ to meet the gain requirement of RF applications happens at the cost of SCEs and with a tall $H_{\text {fin }}$, which also induces difficulties in manufacturing process. Alternatively, the multi-fin configuration constructed with a number of fingers $\left(N_{\text {finger }}\right)$ and multiple fins per fingers $\left(N_{\text {fin }}\right)$ shows a practical means to ease above mentioned concerns. The total number $\left(N_{\text {FinFET }}=N_{\text {finger }} \times N_{\text {fin }}\right)$ of FinFETs in such a structure usually is large, i.e., several hundreds of transistors [2]. Considering the complexity and realistic time limit of simulations in TCAD, we have simplified the structure in [2] by setting $N_{\text {finger }}=1$ as shown in Figure 2. The spacing between each fin is set as $50 \mathrm{~nm}$ to suit the $22 \mathrm{~nm}$ node technology and to achieve optimum RF characteristics [8]. Results in Figure 3(a)

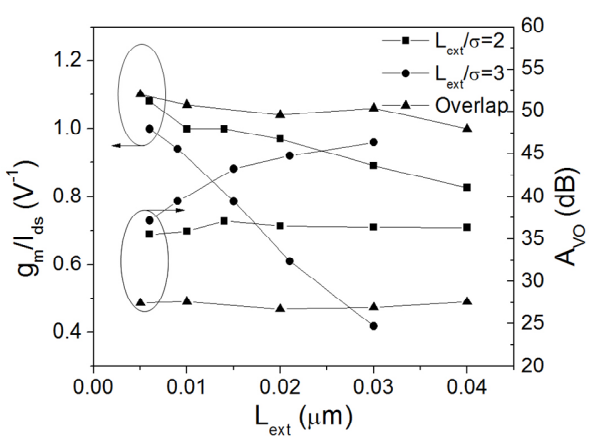

(a)

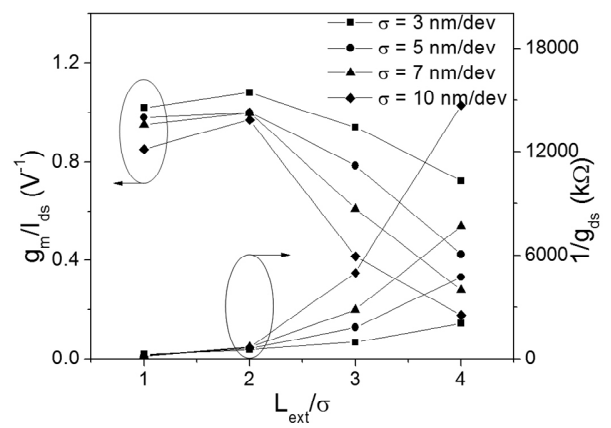

(b)

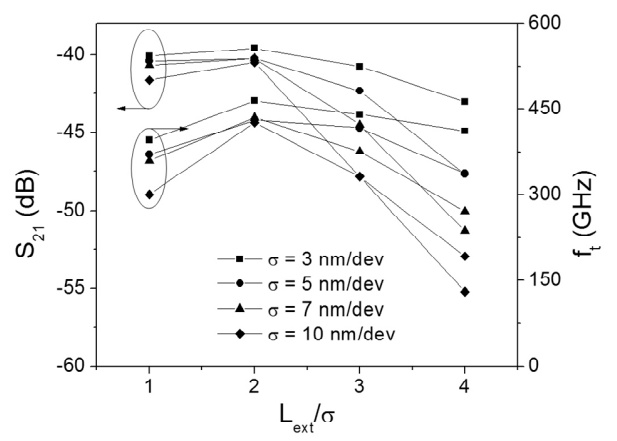

(c)

Figure 1. (a) Variation of $g_{\mathrm{m}} / I_{\mathrm{ds}}$ and $A_{\mathrm{vo}}$ versus $L_{\mathrm{ext}}$; (b) Variation of $g_{\mathrm{m}} / I_{\mathrm{ds}}$ and $1 / g_{\mathrm{ds}}$; and (c) $S_{21}$ and $f_{\mathrm{t}}$ versus $L_{\text {ext }} / \sigma_{\mathrm{S} / \mathrm{D}}$ ratio extracted at $\mathrm{I}_{\mathrm{ds}}=100 \mu \mathrm{A} / \mu \mathrm{m}$ for various $\sigma_{\mathrm{S} / \mathrm{D}}$ value. Device parameters: $W_{\mathrm{fin}}=12 \mathrm{~nm}$, and $V_{\mathrm{ds}}=1.0 \mathrm{~V}$.

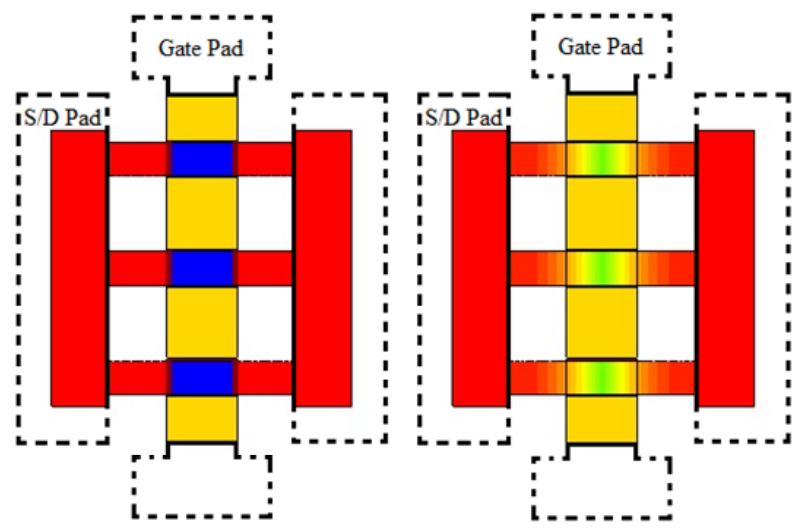

Figure 2. Schematic diagram of overlap and underlap multifin structure analyzed in this brief. 


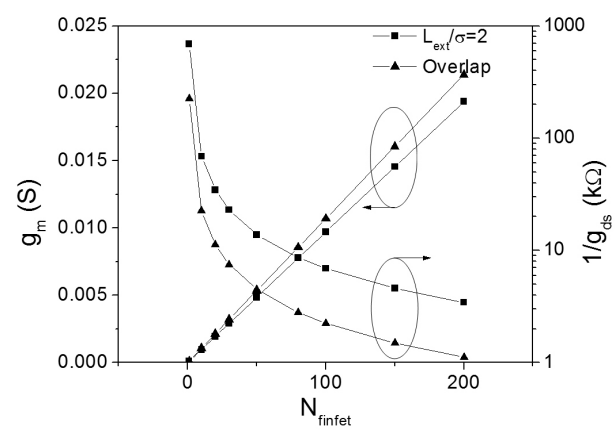

(a)

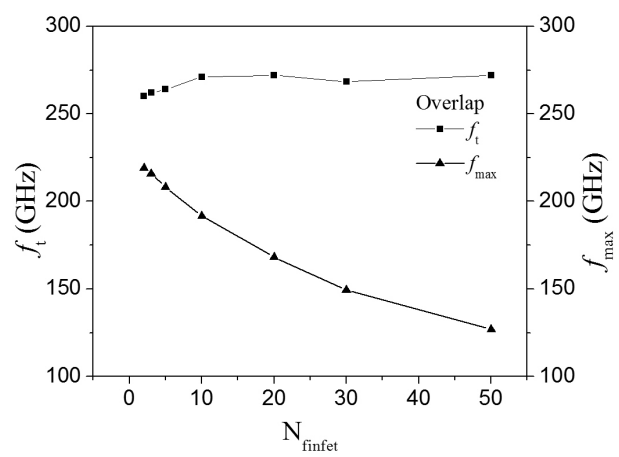

(b)

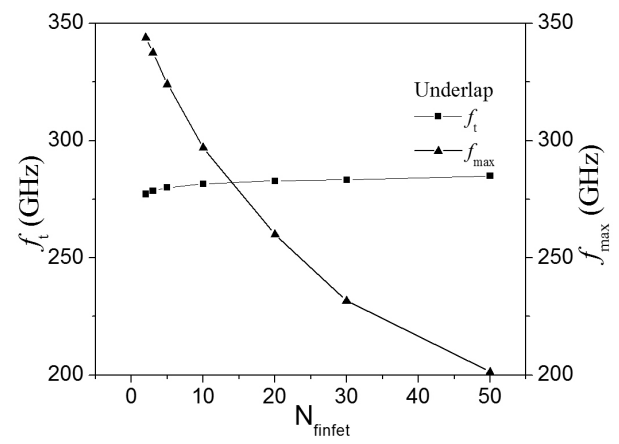

(c)

Figure 3. (a) Variation of $g_{\mathrm{m}}$ and $1 / g_{\mathrm{ds}}$ extracted at $\mathbf{I}_{d s}=100$ $\mu \mathrm{A} / \mu \mathrm{m}$ as a function of $N_{\text {FinFET }}$ at $10 \mathrm{~Hz}$; and $f_{\mathrm{t}}$ and $f_{\text {max }}$ of (b) Overlap and (c) Underlap structure extracted at $V_{\mathrm{gs}}=0.6 \mathrm{~V}$. Device parameters: $W_{\text {fin }}=12 \mathrm{~nm}$, and $V_{\mathrm{ds}}=1.0 \mathrm{~V}$.

show a good linearity of the intrinsic $g_{\mathrm{m}}$ versus $N_{\text {FinFET }}$ for both underlap and overlap design models. It should be noted that the $A_{\text {vo }}$ for multi-fin FETs does not benefit from arrays of transistors. It is limited to the base FinFET analog FoM because of the equation that $A_{\mathrm{vo}}=$ $N_{\text {FinFET }} \cdot g_{\mathrm{m}} / N_{\text {FinFET }} \cdot g_{\text {ds }}=g_{\mathrm{m}} / g_{\text {ds }}$. This can be verified by the degra- dation of $1 / g_{\mathrm{ds}}$ as shown in Figure 3(a).

The RF FoM $f_{\mathrm{t}}$ and $f_{\max }$ are simulated using the TCAD mixed-mode module with considerations of parasitic resistances and capacitances associated with gate pads, S/D contacts and coupling effects. Those extrinsic components are identified as the bottleneck of the RF performance and the values depending on process techniques. In the simulated structure, the parasitic gate capacitance is mainly due to the fringe capacitance $C_{\mathrm{f}}$ which is set at $0.18 \mathrm{fF} / \mu \mathrm{m}$ as per ITRS[1]. For a multi-fin FET, $C_{\text {fmulti }}$ is almost linearly proportional to $N_{\text {FinFET }}$ [2] and therefore for simulation simplification purpose we can conclude that

$$
C_{\text {fmulti }}=N_{\text {FinfET }} \cdot C_{\mathrm{f}} .
$$

The normalized series resistance $R_{\mathrm{SD}}$ is taken the value of $250 \Omega \cdot u m$ [1] and the S/D contact pad of single-fin transistor is designed as $0.04 \times 0.06 \mathrm{um}^{2}$. As the fin number increased, the spacing between each fin will be covered with contact and we can model the multi-fin structure $\mathrm{S} / \mathrm{D}$ extrinsic series resistance as:

$$
R_{\mathrm{SDmulti}}=R_{\mathrm{SD}} \times\left[6 /\left(7 \cdot N_{\mathrm{FinFET}}-1\right)\right]
$$

The parasitic gate resistance for a single fin is set as four times of the $R_{\mathrm{SD}}$, due to the limited contact area, to be $1000 \Omega \cdot u m$, including a $50 \Omega \cdot$ um for the gate pad component [2]. Therefore, for multi-fin, the gate extrinsic resistance will be:

$$
R_{\text {gmulti }}=50+950 / N_{\text {FinFET }}
$$

Including the parasitic components, the simulated RF FoM is shown in Figures 3(b) and 3(c). For $N_{\text {FinFET }}=2$, the overlap structure shows $f_{\mathrm{t}}$ of $260 \mathrm{GHz}$ and $f_{\max }$ of 219 $\mathrm{GHz}$, which is consistent with the reported result [8]. As $N_{\text {FinfET }}$ increases, $f_{\mathrm{t}}$ does not vary a lot according to the equation that

$$
f_{\mathrm{t}} \approx g_{\mathrm{m}} /\left[2 \pi \cdot\left(C_{\text {ggintrinsic }}+C_{\mathrm{f}}\right)\right]
$$

where the transconductance and the capacitances share the same factor $N_{\text {FinFET }}$. However, $f_{\max }$ starts to degrade because the large component $R_{\text {gmulti }}$ is non-linearly inversely proportional to $N_{\text {FinFET }}$ and this leads to the decrease of $f_{\max }$ based on the equation in [9]. The $f_{\max }$ in multi-fin FETs with overlap design drops below 200 $\mathrm{GHz}$ as $N_{\text {FinFET }}$ goes only beyond 10 . In contrast, the underlap design, shows a comparable $f_{\mathrm{t}}$ and a much higher $f_{\max }$, compared to the corresponding overlap design. The higher $f_{\max }$ value is due to the significant reduction in $g_{\mathrm{ds}}$ (Figure 3(a)) [8]. As $N_{\text {FinFET }}$ increases, although underlap structure shows degradation in $f_{\max }$ as overlap one, it still maintain the maximum frequency and cutoff frequency above $200 \mathrm{GHz}$ even when $N_{\text {fin }}$ reaches 50 .

\section{Conclusion}

It is shown that the multi-fin FET will be particularly useful at sub-32 $\mathrm{nm}$ regime for the development of devices for RF applications. Even with a large number of $N_{\text {FinFET, }}$, using underlap design in SDE region with an optimal value of $L_{\text {ext }} / \sigma_{\mathrm{S} / \mathrm{D}}$ ratio, good SCE is achieved and its RF FoM $f_{\mathrm{t}}$ and $f_{\text {max }}$ are better than the one with overlap design. Furthermore, the cost of multi-fin device is expected to be lower than other heterojunction devices 
and III-V compound devices because of its compatibility with the CMOS planar process technology.

\section{Acknowledgements}

The authors would like to thank Synopsys, Inc., for providing the Sentaurus TCAD tool set for device design and simulation.

\section{REFERENCES}

[1] "International Technology Roadmap for Semiconductors," 2011. http://www.public.itrs.net

[2] V. Subramanian, A. Mercha, B. Parvais, M. Dehan, G. Groeseneken, W. Sansen and S. Decoutere, "Identifying the Bottlenecks to the RF Performance of FinFETs," Proceedings of the 23rd Annual International Conference on VLSI Design, Bangalore, 3-7 January 2010, pp. 111-116.

[3] V. Trivedi, J. G. Fossum and M. M. Chowdhury, "Nanoscale FinFETs with Gate-Source/Drain Underlap," IEEE Transactions on Electron Devices, Vol. 52, No. 1, 2005, pp. 56-62. doi:10.1109/TED.2004.841333

[4] A. Kranti and G. M. Armstrong, "Design and Optimization of FinFETs for Ultra-Low-Voltage Analog Applications," IEEE Transactions on Electron Devices, Vol. 54,
No. 12,2007 , pp. 3308-3316. doi:10.1109/TED.2007.908596

[5] J. Yang, P. M. Zeitzoff and H. Tseng, "Highly Manufacturable Double-Gate FinFET With Gate-Source/Drain Underlap," IEEE Transactions on Electron Devices, Vol. 54, No. 6, 2007, pp. 1464-1470. doi:10.1109/TED.2007.896387

[6] P. Feng and P. K. Ghosh, "Comparison of Silicon-onInsulator and Body-on-Insulator FinFET Based Digital Circuits with Consideration on Self-Heating Effects," IEEE International Semiconductor Device Research Symposium, College Park, Maryland, 7-9 December 2010, pp. $1-2$.

[7] “Sentaurus Device Manual," Synopsys, Santa Clara, 2010.

[8] A. Kranti, J. Raskin and G. A. Armstrong, "Optimizing FinFET Geometry and Parasitics for RF Applications," Proceedings of the IEEE International SOI Conference, New Paltz, New York, 6-9 Octorber 2008, pp. 123-124.

[9] J.-P. Raskin, G. Pailloncy, D. Lederer, F. Danneville, G. Dambrine, S. Decoutere, A. Mercha and B. Parvais, "HighFrequency Noise Performance of 60-nm Gate-Length FinFETs," IEEE Transactions on Electron Devices, Vol. 55, No. 10, 2008, pp. 2718-2727. doi:10.1109/TED.2008.2003097 